\title{
A systematic review and meta-analysis of the pronator quadratus repair following volar plating of distal radius fractures
}

\author{
Chun-Kuan Lu, ${ }^{1,2}$, Wen-Chih Liu' ${ }^{1,2,3^{*}} \mathbb{D}$, Chung-Chia Chang ${ }^{1}$, Chia-Lung Shih ${ }^{1}$, Yin-Chih Fu ${ }^{1,4}$ and Jesse B. Jupiter ${ }^{5}$
}

\begin{abstract}
Background: Distal radius fracture (DRF) is the most common upper extremity fracture that requires surgery. Operative treatment with a volar locking plate has proved to be the treatment of choice for unstable fractures. However, no consensus has been reached about the benefits of pronator quadratus (PQ) repair after volar plate fixation of DRF in terms of patient-reported outcome measures, pronation strength, and wrist mobility.
\end{abstract}

Methods: We searched the PubMed, Embase, Cochrane Central, and China National Knowledge Infrastructure (CNKI) databases up to March 13, 2020, and included randomized-controlled, non-randomized controlled, or case-control cohort studies that compared cases with and without PQ repair after volar plate fixation of DRF. We used a randomeffects model to pool effect sizes, which were expressed as standardized mean differences (SMDs) and 95\% confidence intervals. The primary outcomes included Disabilities of the Arm, Shoulder, and Hand scores and pronation strength. The secondary outcomes included the SMDs in pain scale score, wrist mobility, and grip strength. The outcomes measured were assessed for publication bias by using a funnel plot and the Egger regression test.

Results: Five randomized controlled studies and six retrospective case-control studies were included in the metaanalysis. We found no significant difference in primary and secondary outcomes at a minimum of 6-month follow-up. In a subgroup analysis, the pronation strength in the PQ repair group for $A O$ type $B$ DRFs (SMD $=-0.94 ; 95 \% \mathrm{Cl},-1.54$ to $-0.34 ; p<0.01)$ favored $\mathrm{PQ}$ repair, whereas that in the PQ repair group for non-AO type B DRFs $(S M D=0.39 ; 95 \% \mathrm{Cl}$, $0.07-0.70 ; p=0.02$ ) favored no PQ repair.

Discussion: We found no functional benefit of PQ repair after volar plate fixation of DRF on the basis of the present evidence. However, PQ muscle repair showed different effects on pronation strength in different groups of DRFs. Future studies are needed to confirm the relationship between PQ repair and pronation strength among different patterns of DRF.

Registration: This study was registered in the PROSPERO registry under registration ID No. CRD42020188343. Level of evidence: Therapeutic III

Keywords: Pronator quadratus, Distal radius fracture, Volar plate, Pronation

\footnotetext{
* Correspondence: andysirliu@gmail.com

'Department of Orthopedic Surgery, Kaohsiung Medical University Hospital, Kaohsiung Medical University, Kaohsiung, Taiwan

${ }^{2}$ Ph.D Program in Biomedical Engineering, College of Medicine, Kaohsiung Medical University, Kaohsiung, Taiwan

Full list of author information is available at the end of the article
}

C C The Author(s). 2020 Open Access This article is licensed under a Creative Commons Attribution 4.0 International License, which permits use, sharing, adaptation, distribution and reproduction in any medium or format, as long as you give appropriate credit to the original author(s) and the source, provide a link to the Creative Commons licence, and indicate if changes were made. The images or other third party material in this article are included in the article's Creative Commons licence, unless indicated otherwise in a credit line to the material. If material is not included in the article's Creative Commons licence and your intended use is not permitted by statutory regulation or exceeds the permitted use, you will need to obtain permission directly from the copyright holder. To view a copy of this licence, visit http://creativecommons.org/licenses/by/4.0/ The Creative Commons Public Domain Dedication waiver (http://creativecommons.org/publicdomain/zero/1.0/) applies to the data made available in this article, unless otherwise stated in a credit line to the data. 


\section{Introduction}

Distal radius fracture (DRF) is the most common upper extremity fracture that requires surgery. Operative treatment with a volar locking plate has proved to be the treatment of choice for unstable fractures [1]. The pronator quadratus (PQ) muscle resides in the fracture zone and implant placement site. Several published studies have addressed methods of preservation or repair of the PQ muscle [2-4]. A previous study surveyed all active members of the American Society for Surgery of the Hand in the USA, and 83\% (608/753) responded that they attempted to repair the PQ muscle [5]. In addition, one previous biomechanical study that included healthy volunteers reported that subjects with decreased pronation torque strength had temporary pronator quadratus paralysis [6]. However, the necessity for repair of the PQ for optimal functional outcome remains controversial.

Two studies, a retrospective case-control study [7] and a prospective randomized controlled study [8], both published in 2013, compared PQ repair with no PQ repair after distal radius plating surgery. They found no significant differences in Disabilities of the Arm, Shoulder, and Hand (DASH) and pain scale scores 1 year postoperatively. An additional prospective randomized controlled study revealed that $P Q$ repair might reduce pain in 3 months postoperatively [9]. The most recently published randomized controlled trial concluded that $\mathrm{PQ}$ repair showed no significant improvement in clinical outcome 1 year after surgery [10].

To compile the best available evidence, we performed a systemic review and meta-analysis of prospective randomized controlled and retrospective case-control studies to determine whether PQ repair after distal radius plating surgery is associated with patient-reported outcome measures, pain scale score, wrist mobility, and grip and pronation strengths.

\section{Methods}

\section{Search strategy and inclusion criteria}

We conducted this study in accordance with the Preferred Reporting Items for Systematic Reviews and Meta-Analyses guidelines [11]. We performed an electronic search in the PubMed, Embase, Cochrane Central, and China National Knowledge Infrastructure (CNKI) databases up to March 13, 2020, using search strategies (Additional file 1 Appendix 1). The bibliographies of the included trials and related review articles were manually reviewed for relevant references. Two independent reviewers (CCC and CKL) evaluated the studies by screening the titles and abstracts, followed by a detailed examination of the full texts of the eligible articles. Any inconsistencies were resolved using a consensual approach. If a disagreement could not be resolved, we consulted a third reviewer (WCL) for the final decision.

Regarding the types of studies included, we enrolled randomized controlled trials (RCTs) and comparative experimental trials. We included clinical trials that met the following criteria: (1) included a target population that was comprised of patients with DRFs treated with volar plate fixation; (2) comprised of two treatment arms after volar plate fixation, with and without PQ repair; and (3) measured the clinical outcome at least 6 months postoperatively. We excluded the following types of studies: (1) reviews, conference abstracts, or presentations, and (2) overlapping publications. We summarized the selection process in accordance with the PRISMA flowchart (Fig. 1).

\section{Methodological quality assessment}

All 12 studies were critically appraised for the assessment of their methodological qualities by two independent reviewers (WCL and CKL), which was checked by a third reviewer (CLS). We recorded the first author, year, number of fracture patterns based on the AO classification, participant characteristics, model of the volar plate, and detailed technique of the PQ repair. The methodological qualities of the enrolled studies were evaluated by two reviewers independently using the Jadad scale for RCTs [12] and the Newcastle-Ottawa Scale (NOS) for comparative experimental trials [13]. The Jadad scale evaluates the methodology of RCTs in accordance with three aspects as follows: randomization (2 points), blinding (2 points), and an account of all patients (1 point) [12]. The five RCTs included in our study ranged had Jadad scale scores ranging from 3 to 5, with a maximum possible score of 5 . Higher scores indicate better methodological quality. In evaluating case-control studies, the NOS contains nine items in three categories as follows: participant selection (four items), comparability (one item), and exposure (three items). A study can be scored a maximum of 1 point for items in the selection and exposure domains and a maximum of 2 points for the comparability domain [14]. The five case-control studies included all had a NOS score of 8 , with a maximum possible score of 9 . Higher scores indicate better methodological quality. Between-reviewer discrepancies were resolved through discussions under the supervision of the corresponding author. We tried to contact the primary authors of all the included studies; however, only the authors of three studies responded and provided complete original data sheets $[7,8,10]$. 


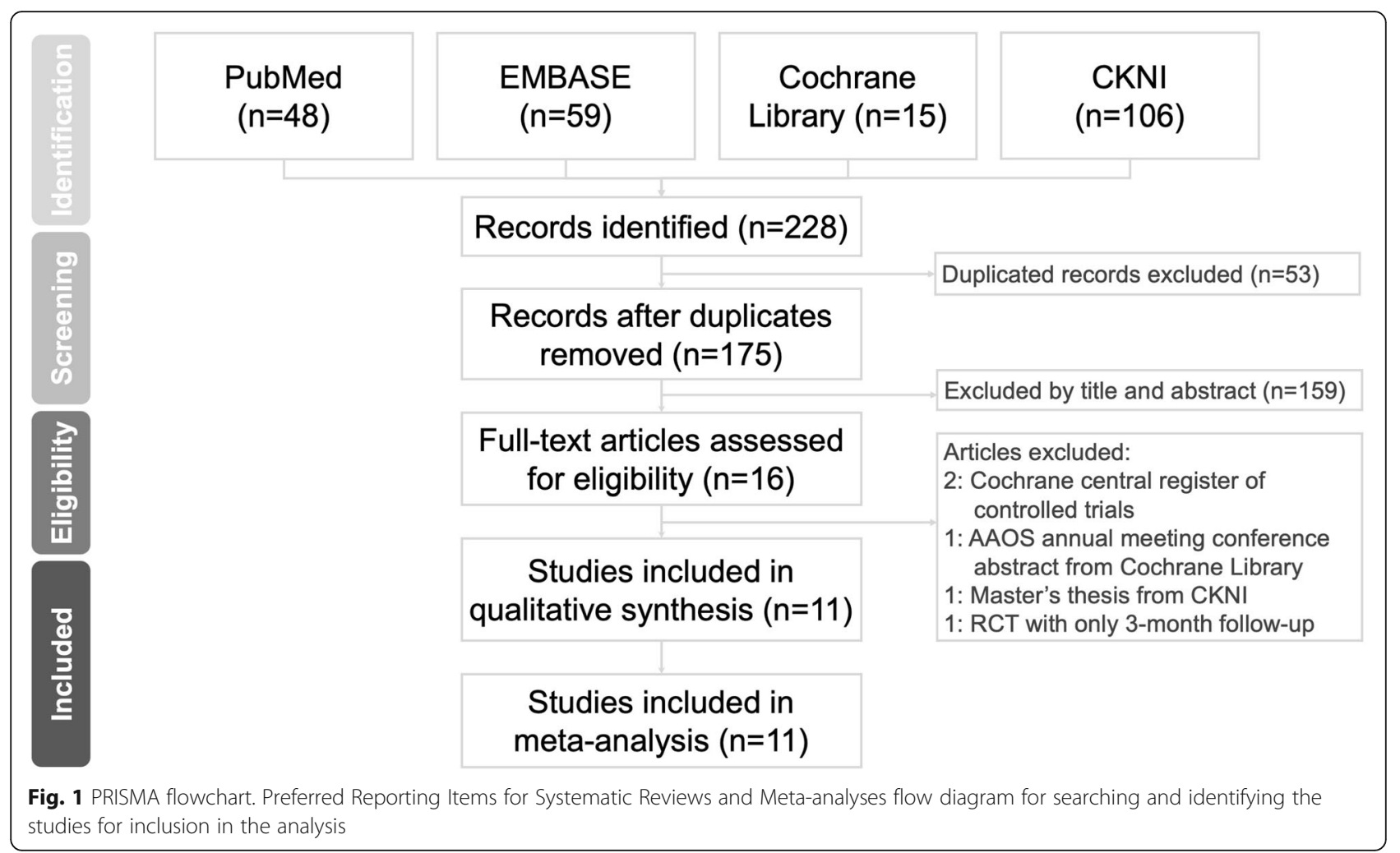

\section{Meta-analysis methodology}

A meta-analysis was performed using the Comprehensive Meta-Analysis (CMA) version 3 software (Biostat, Englewood, NJ, USA), thereby combining the relevant effects of interest from our identified studies.

All the outcomes were assessed within two groups after volar plate fixation of DRF as follows: PQ repair was performed in the study group but not in the control group in a minimum of 6 months after distal radius plating surgery. The standardized mean differences (SMDs) in DASH score and pronation strength between the two groups were the primary outcomes. The SMDs in wrist mobility (extension, flexion, supination, pronation, radial deviation, and ulnar deviation) and grip strength and visual analog scale score for pain between the groups were the secondary outcomes. A negative SMD value indicated that PQ repair was a favorable treatment option. Studies that did not report standard deviations (SDs) were excluded from the pooling. We also recorded any flexor complications mentioned in each included study. When the 95\% confidence interval (CI) of the summary mean did not overlap, we considered it statistically significant. Between-trial heterogeneity was determined using $I^{2}$ and chi-square tests [15]. A fixed-effects (inverse variance) model was used when the effects were assumed to be homogenous $(p>0.01)$. Statistical heterogeneity is implied when the $p$ value was $<0.01$; thus, a random-effects model was used in those circumstances.
Articles that reported outcome measures were assessed for publication bias using a funnel plot [16] and the Egger regression test [17].

\section{Results}

Literature search and study characteristics

We retrieved 175 non-duplicate citations and reviewed their titles and abstracts, and included 15 articles for meticulous evaluation after eliminating references that did not meet the inclusion criteria (Fig. 1). We excluded two studies from the Cochrane Central Register of Controlled Trials database, one master's thesis, which is a review article from the CNKI database, and one RCT that compared PQ repair with no PQ repair with only 3 months of follow-up. Therefore, the meta-analysis included five RCTs $[8,10,18-20]$ and six retrospective case-control studies [7, 21-25].

A total of 732 patients were included along with a breakdown of the numbers of patients, mean ages, and sex ratios in comparison groups, except for two studies. Patient sex data and mean patient age were well recorded in all the studies. The mean ages of the patients ranged from 48.7 to 64.0 years in the group with PQ repair and from 47.1 to 63.6 years in the group without PQ repair. The female-to-male ratio in each study was well proportioned between the two study groups. The mean patient age and sex ratio were also comparable in all the studies. The details of each study are summarized in Table 1. 


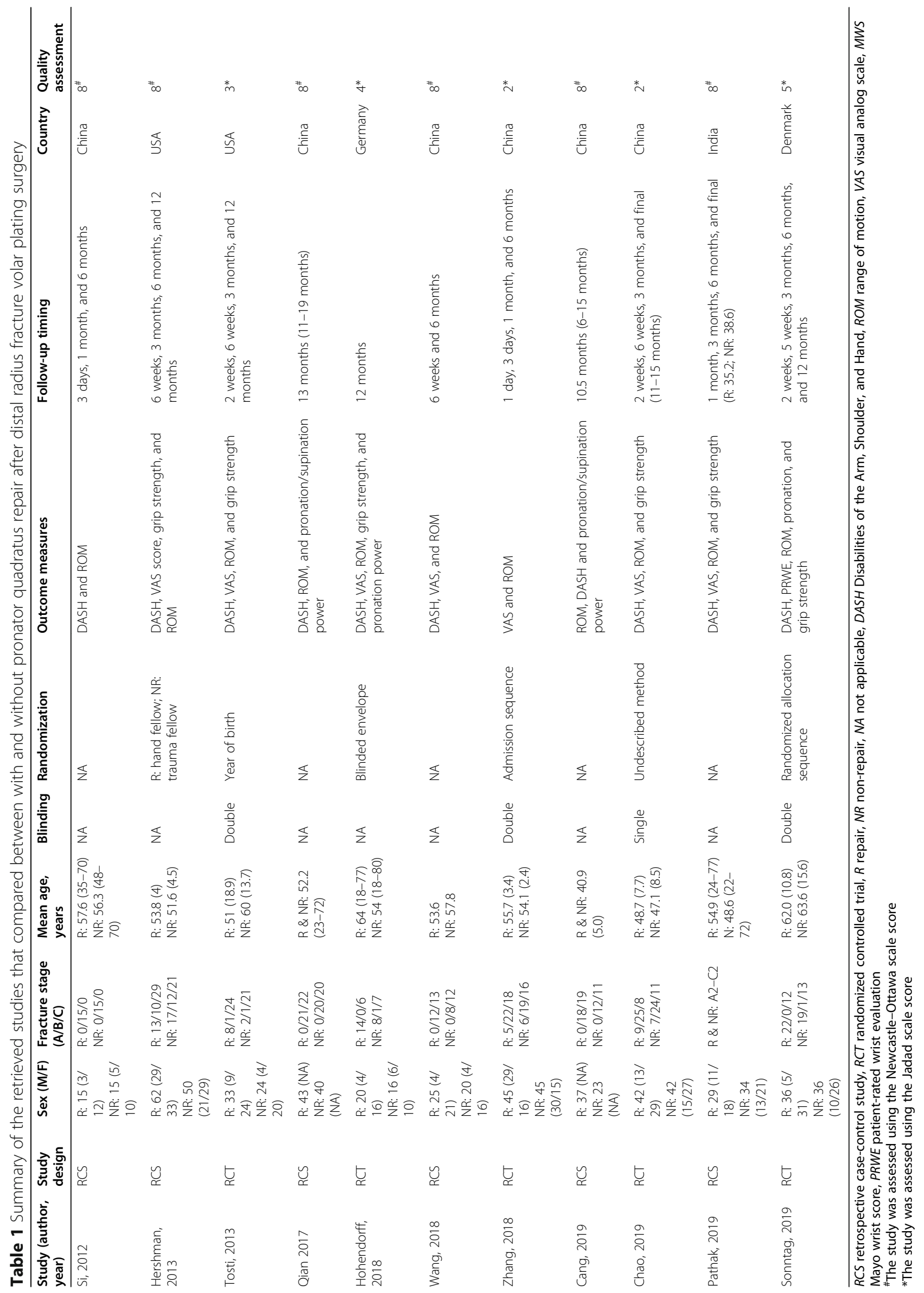


For pronation strength measurement, two studies $[10,18]$ used a baseline hydraulic wrist dynamometer (Fabrication Enterprises), and another two studies [25] used a hand dynamometer (Qianli, China). For grip strength measurement, three studies $[8,10,18]$ used a dynamometer (Jamar; Therapeutic Equipment, Clifton, NJ) and two studies [7, 19] did not mention the hand dynamometer model used. One study [8] provided grip strength data in comparison with those of the uninjured side instead of the actual measured values.

Most studies presented the number of fractures in each AO Foundation/Orthopaedic Trauma Association (AO/OTA) fracture classification [26], except one study [23] that did not report the included fracture types. Two studies analyzed the outcomes of patients with DRF AO/OTA types B and C [22, 25] with or without PQ repair instead of the pooled outcomes. We divided these two studies into two sub-studies for the statistical analysis.

\section{Surgical procedure and postoperative management}

All the internal fixation surgeries were performed using the modified volar approach of Henry. Among the included studies, different types of volar plate, methods of PQ repair, and postoperative management strategies were used. We summarized them in Table 2.

\section{Meta-analysis of the outcomes}

We retrieved the long-term follow-up outcome from each study. Figures 2 and 3 show the forest plot of the different clinical outcomes between the two groups. We demonstrated the pooled mean of the outcomes in Table 3. No significant difference in each outcome was found between the two treatment arms. In a subgroup analysis of DRFs of AO type B or nontype $B$, we found a significant difference in pronation strength between the two treatment arms $(\mathrm{SMD}=$ $0.94 ; 95 \% \mathrm{CI},-1.54$ to $-0.34 ; p<0.01$, favoring PQ repair in the type $\mathrm{B}$ group vs $\mathrm{SMD}=0.39 ; 95 \% \mathrm{CI}$, $0.07-0.70 ; p=0.02$, favoring $\mathrm{PQ}$ repair in the nontype B group; Fig. 4).

The Egger test revealed no significant publication bias regarding most clinical outcomes except radial deviation range of motion $(t=39.2, d f=1, p=0.02$; Table 4$)$.

\section{Discussion}

With the popularity of the volar locking plate in the management of unstable DRFs, demonstrable repair of the PQ muscle postoperatively has become an issue. The PQ consists of a superficial head, which acts on forearm pronation, and a deep head, which is a dynamic stabilizer of the distal radioulnar joint [27]. As noted, a clinical study in healthy volunteers demonstrated that pronation strength decreased by $21 \%$ after the PQ muscle was anesthetized [6]. However, most clinical studies that compared cases with and without PQ repair after distal radius volar plating surgery did not show this significant difference.

Regarding pronation strength, two studies showed that the pronation strength in the group without PQ repair decreased significantly only in the patients with $\mathrm{AO} /$ OTA type B fractures but not in those with AO/OTA

Table 2 Summary of the surgery and postoperative management details of the retrieved studies

\begin{tabular}{|c|c|c|c|}
\hline $\begin{array}{l}\text { Study (author, } \\
\text { year) }\end{array}$ & Plate & $P Q$ repair method & Postoperative management \\
\hline Si, 2012 & Unknown VLP & Direct interrupted (absorbable 2-0) & Physical therapy in 2 days \\
\hline Hershman, 2013 & Stryker and Synthes VLP & NA & Splint for 2 weeks, full weight bearing in 6 weeks \\
\hline Tosti, 2013 & $\begin{array}{l}\text { Medartis (VA) and Synthes } \\
\text { (VA) VLP }\end{array}$ & Figure-of-eight (Vicryl 2-0) & $\begin{array}{l}\text { Immobilization for } 2 \text { weeks and then start of } \\
\text { physical therapy }\end{array}$ \\
\hline Qian 2017 & Synthes VLP & Figure-of-eight (Vicryl 3-0) & NA \\
\hline Hohendorff, 2018 & Stryker (VA) VLP & PQ to BR (PDS 4-0) & $\begin{array}{l}\text { Splint for } 2 \text { weeks and unlimited mobility in } 4 \\
\text { weeks }\end{array}$ \\
\hline Wang, 2018 & NA & Figure-of-eight (absorbable 4-0) & Physical therapy in 2 days \\
\hline Zhang, 2018 & Unknown VLP & Direct interrupted (absorbable 2-0) & Physical therapy in 2 days \\
\hline Cang, 2019 & Synthes VLP & Figure-of-eight (absorbable 3-0) & NA \\
\hline Chao, 2019 & NA & Direct interrupted (absorbable 3-0) & Physical therapy in 1 weeks \\
\hline Pathak, 2019 & NA & Direct interrupted (Vicryl 3-0) & $\begin{array}{l}\text { Immobilization for } 1-2 \text { weeks and then start of } \\
\text { physical therapy }\end{array}$ \\
\hline Sonntag, 2019 & Stryker (VA), Synthes (VA) & $\begin{array}{l}\text { Continuous with a minimum of four sutures } \\
\text { (Vicryl 3-0) }\end{array}$ & Splint for 2 weeks and gradual weight bearing \\
\hline
\end{tabular}




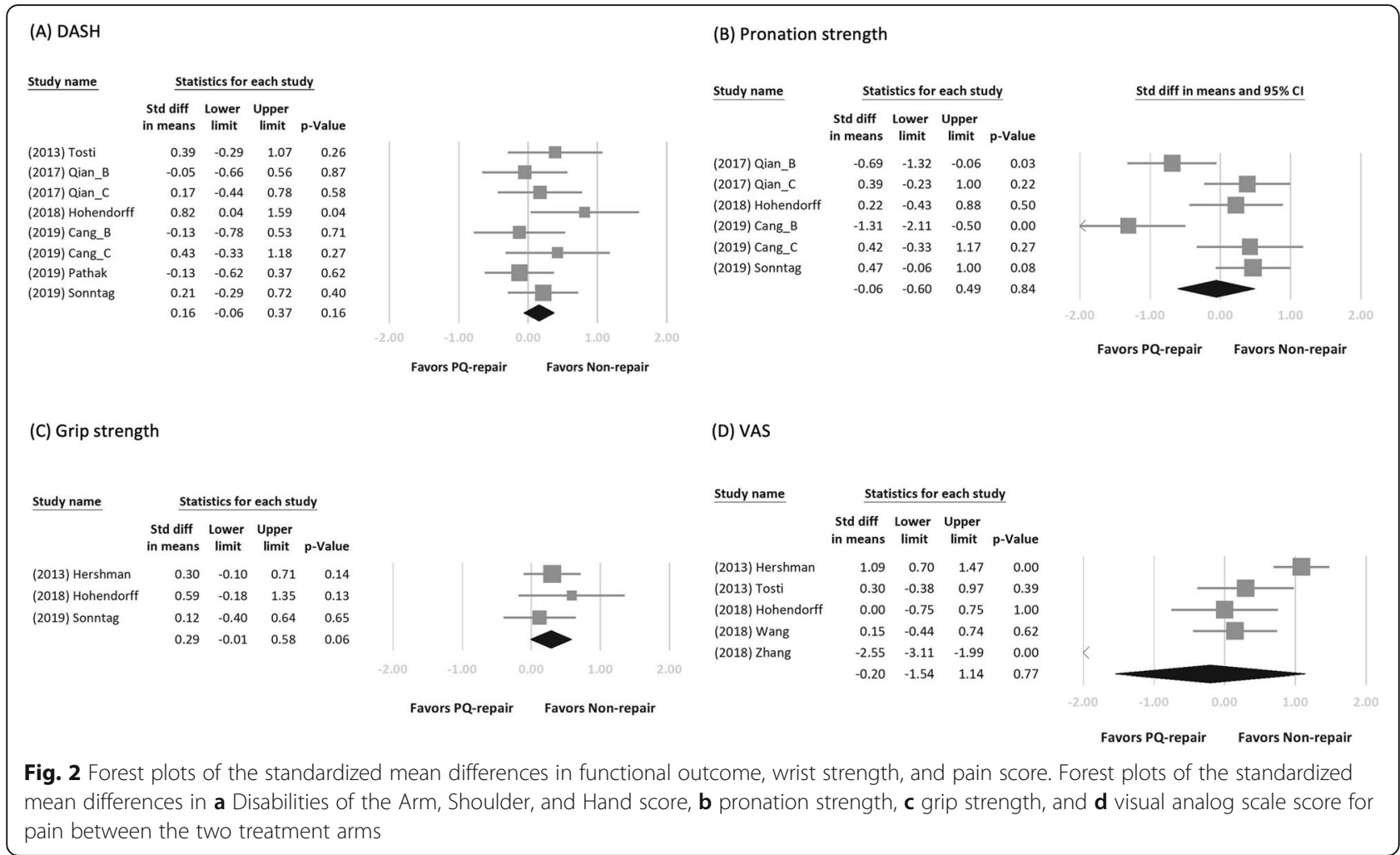

type $C$ fractures [22, 25]. One study included patients with AO/OTA type A2, A3, and $\mathrm{C} 1$ fractures [18], while another study included patients with AO/OTA type A2, A3, C1-3 fractures [10]. Having better repair quality with preoperatively intact and nicely prepared PQ flaps seems logical. Although the durability of PQ repair has been supported [5], ensuring good-quality repair, especially in comminuted DRFs with frayed PQ muscles, is sometimes difficult. Two RCTs routinely checked the length or retraction of the PQ in ultrasonography examinations $[10,18]$. In our included studies, patients in the group without PQ repair who had more metaphyseal displaced and complex fracture patterns had better pronation strengths (Fig. 4). However, a retrospective study showed that the completeness of PQ repair did not influence wrist mobility and grip strength [28]. More evidence is needed to confirm the relationship between the quality of PQ repair and pronation strength among different patterns of DRF.

The reliability and validity of the DASH questionnaire in the German, Chinese, and Danish versions have been confirmed [29-31]. Fracture classification might influence clinical outcomes. We found that most studies included patients with similar distributions of AO/OTA types $\mathrm{A}, \mathrm{B}$, and $\mathrm{C}$. As a result, this factor was controlled within each group. The other factor is postoperative treatment. Each study had a different postoperative treatment. An RCT found that patients starting wrist mobilization 2 or 6 weeks after volar plate fixation of the distal radius did not influence the patient-reported outcome measures, grip strength, and wrist mobility at 6 and 12 months [32]. Although each study used varied postoperative treatments, we could assume that it did not influence the postoperative outcome in a minimum of 6 months for quantitative calculation.

This systematic review is limited by the sample size and quality of the available studies. To compensate for limiting the research articles in English, we conducted an extensive search strategy in most available databases, including CNKI, to ensure that all potentially relevant papers were identified and reviewed. The measurement tools for grip and pronation strength varied greatly in the included studies, which could influence the data quantification. Finally, three authors provided raw data, making up for the missing items in the included studies $[7,8,10]$.

One potential benefit of PQ repair is that it enables the separation of the volar plate from the flexor tendon, which might prevent complications such as flexor tendon irritation or rupture. A systematic review revealed that the median interval between surgery and flexor 


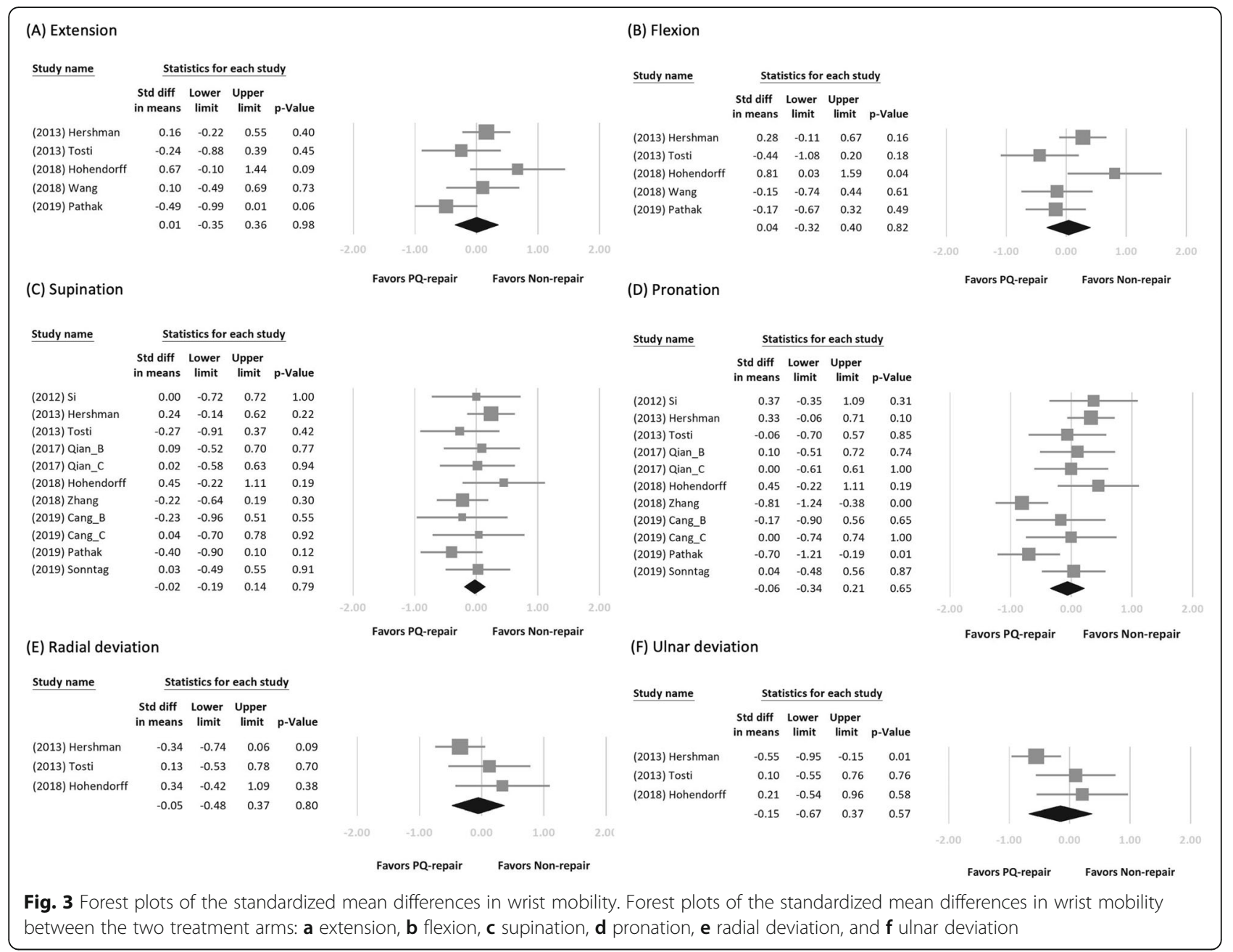

Table 3 Summary of the outcomes by meta-analysis

\begin{tabular}{|c|c|c|c|c|c|c|}
\hline & \multicolumn{3}{|l|}{ With $P Q$ repair } & \multicolumn{3}{|l|}{ Without $P Q$ repair } \\
\hline & Pooled estimate & $95 \% \mathrm{Cl}$ & $n$ & Pooled estimate & $95 \% \mathrm{Cl}$ & $n$ \\
\hline$\overline{D A S H}$ & 24.73 & $16.22-33.25$ & $8^{*}$ & 22.15 & $9.86-34.44$ & $8^{*}$ \\
\hline Pronation strength $(\mathrm{kg} \mathrm{cm})$ & 50.70 & $44.32-67.08$ & $6^{*}$ & 57.39 & $40.32-74.45$ & $6^{*}$ \\
\hline Grip strength $(\mathrm{kg})$ & 32.63 & $19.86-45.39$ & 3 & 32.86 & $19.77-45.94$ & 3 \\
\hline VAS & 1.39 & $0.89-1.89$ & 5 & 1.61 & $0.40-2.81$ & 5 \\
\hline Extension (degrees) & 62.84 & $45.09-80.59$ & 6 & 63.94 & $52.31-75.57$ & 6 \\
\hline Flexion (degrees) & 62.18 & $48.99-75.36$ & 6 & 63.23 & $53.05-73.41$ & 6 \\
\hline Supination (degrees) & 68.12 & $53.82-82.42$ & $11^{*}$ & 68.66 & $53.63-83.69$ & $11^{*}$ \\
\hline Pronation (degrees) & 62.86 & $52.24-73.24$ & $11^{*}$ & 63.00 & $52.36-73.64$ & $11^{*}$ \\
\hline Radial deviation (degrees) & 19.07 & $16.52-21.62$ & 3 & 20.67 & $18.95-22.39$ & 3 \\
\hline Ulnar deviation (degrees) & 31.42 & $25.60-37.25$ & 3 & 33.98 & $31.45-36.51$ & 3 \\
\hline
\end{tabular}

PQ pronator quadratus, SMD standardized mean difference, $\mathrm{Cl}$ confidence interval, DASH Disabilities of the Arm, Shoulder, and Hand, VAS visual analog scale for pain

*Presented outcomes separately for AO type B and C fractures, which we counted as two studies 


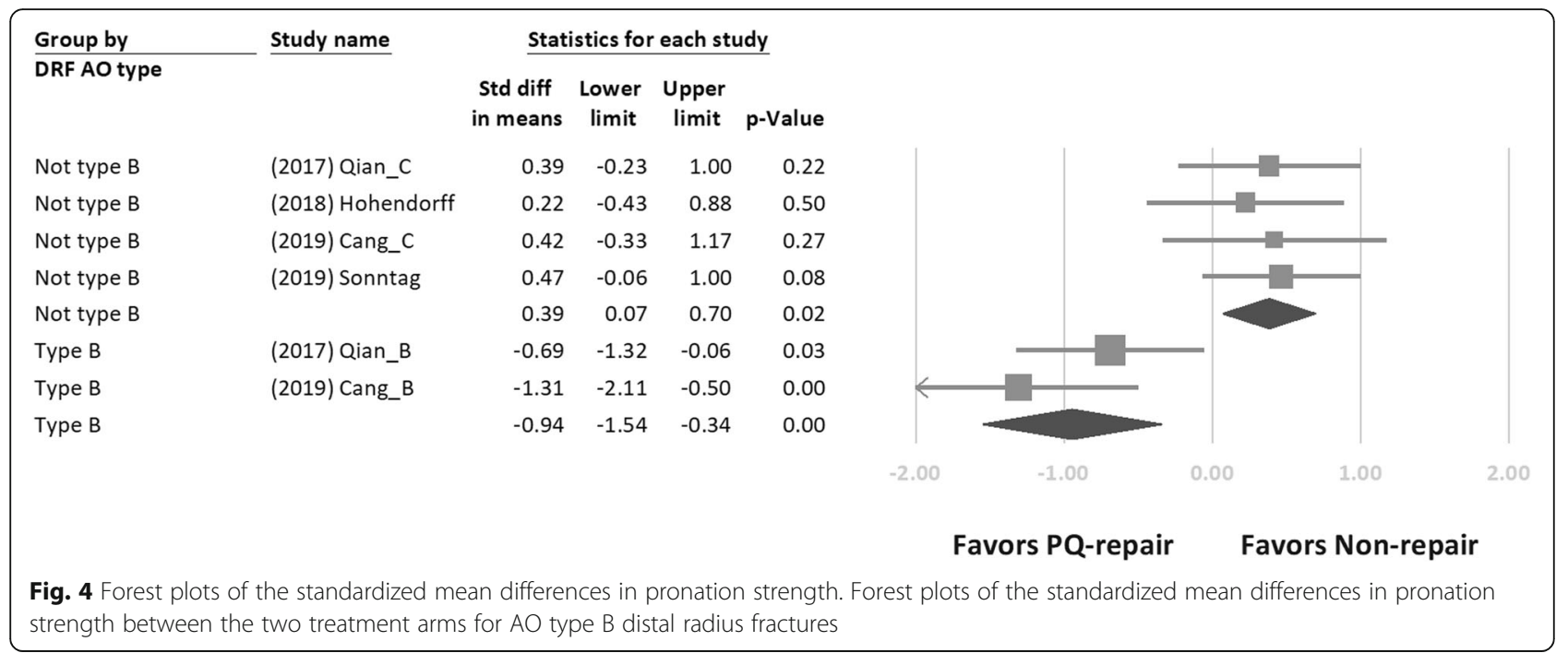

tendon rupture was 9 months (interquartile range, 6-26 months) [33]. A cohort study included 451 patients with a mean follow-up of 3.2 years. The flexor tendon rupture rate was only $1.1 \%$ [34]. In the studies included in our analysis, no flexor tendon rupture was found in each group. The discrepancy in the literature may be attributed to the difference in follow-up duration. We could not conclude whether PQ repair is a protective factor against flexor complications. Future studies with longer follow-up periods are needed to compare between with and without PQ repair.

\section{Conclusion}

This systematic review and meta-analysis found no significant differences between the groups with and without PQ repair in terms of DASH score, pronation strength, pain score, wrist mobility, and grip strength at a

Table 4 Results of the Egger test for each outcome

\begin{tabular}{llll}
\hline & $\boldsymbol{t}$ & $\boldsymbol{d f}$ & $\boldsymbol{p}$ value \\
\hline DASH & 2.08 & $6^{*}$ & 0.082 \\
VAS & 0.15 & 3 & 0.891 \\
Extension & 0.27 & 3 & 0.802 \\
Flexion & 0.09 & 3 & 0.931 \\
Supination & 0.05 & $9^{*}$ & 0.958 \\
Pronation & 0.70 & $9^{*}$ & 0.500 \\
Radial deviation & 39.2 & 1 & 0.016 \\
Ulnar deviation & 9.29 & 1 & 0.068 \\
Grip strength & 0.64 & 1 & 0.637 \\
Pronation strength & 1.36 & $4^{*}$ & 0.244 \\
\hline
\end{tabular}

DASH Disability of Disabilities of the Arm, Shoulder, and Hand score, VAS visual analog scale

*Presented outcomes separately for AO type B and C fractures, which we counted as two studies minimum of 6-month follow-up after volar plating surgery for DRF. However, PQ muscle repair showed different effects on pronation strength among the different DRF groups. Future studies are needed to confirm the relationship between PQ repair and pronation strength among the different DRF patterns.

\section{Supplementary information}

Supplementary information accompanies this paper at https://doi.org/10. 1186/s13018-020-01942-w.

Additional file 1:. Search query

\section{Abbreviations}

AO/OTA: AO Foundation/Orthopaedic Trauma Association; Cl: Confidence interval; DASH: Disability of Disabilities of the Arm, Shoulder, and Hand score: DRF: Distal radius fracture; NOS: Newcastle-Ottawa Scale; PQ: Pronator quadratus; RCT: Randomized controlled trial; SMD: Standardized means difference; VAS: Visual analog scale for pain

\section{Acknowledgements}

The authors thank Rick Tosti, Stuart Hershman, Igor Immerman, and Jesper Sonntag for providing us their original data sheets for the completion of this meta-analysis. We appreciate Chih-Wei Hsu for his support in reviewing the structure of this project as well.

Authors' contributions

Each author is expected to have made substantial contributions to the conception. WCL designed of the work; CCC and CLS made the acquisition and analysis; CLS made the data interpretation; CKL and WCL have drafted the work; and YCF and JBJ substantively revised it. The authors read and approved the final manuscript.

\section{Funding}

The authors received no financial support for the research, authorship, and/ or publication of this article.

\section{Availability of data and materials}

The datasets used/or analyzed during the current study are available from the corresponding author on reasonable request.

Ethics approval and consent to participate Not applicable 


\section{Consent for publication}

Not applicable

\section{Competing interests}

The authors declared no potential conflicts of interest with respect to the research, authorship, and/or publication of this article.

\section{Author details}

${ }^{1}$ Department of Orthopedic Surgery, Kaohsiung Medical University Hospital, Kaohsiung Medical University, Kaohsiung, Taiwan. ${ }^{2}$ Ph.D Program in Biomedical Engineering, College of Medicine, Kaohsiung Medical University, Kaohsiung, Taiwan. ${ }^{3}$ Department of Orthopedic Surgery, Kaohsiung Municipal Siaogang Hospital, Kaohsiung Medical University, Kaohsiung, Taiwan. ${ }^{4}$ School of Medicine, College of Medicine, Kaohsiung Medical University, Kaohsiung, Taiwan. ${ }^{5}$ Hand and Upper Extremity Service, Department of Orthopedic Surgery, Massachusetts General Hospital, Harvard Medical School, Boston, MA, USA.

\section{Received: 4 August 2020 Accepted: 1 September 2020}

\section{Published online: 16 September 2020}

\section{References}

1. Saving J, et al. External fixation versus volar locking plate for unstable dorsally displaced distal radius fractures-a 3-year follow-up of a randomized controlled study. J Hand Surg Am. 2019;44(1):18-26. https://doi.org/10.1016/ j.jhsa.2018.09.015.

2. Galmiche C, et al. Minimally invasive plate osteosynthesis for extra-articular distal radius fracture in postmenopausal women: longitudinal versus transverse incision. J Wrist Surg. 2019;8(1):18-23. https://doi.org/10.1055/s0038-1667305.

3. Lee DY, Park YJ, Park JS. A meta-analysis of studies of volar locking plate fixation of distal radius fractures: conventional versus minimally invasive plate osteosynthesis. Clin Orthop Surg. 2019;11(2):208-19. https://doi.org/10. 4055/cios.2019.11.2.208.

4. Cannon TA, et al. Pronator-sparing technique for volar plating of distal radius fractures. J Hand Surg Am. 2014;39(12):2506-11. https://doi.org/10. 1016/j.jhsa.2014.09.011.

5. Swigart CR, et al. Assessment of pronator quadratus repair integrity following volar plate fixation for distal radius fractures: a prospective clinical cohort study. J Hand Surg Am. 2012;37(9):1868-73. https://doi.org/10.1016/j. jhsa.2012.06.005.

6. McConkey $\mathrm{MO}$, et al. Quantification of pronator quadratus contribution to isometric pronation torque of the forearm. J Hand Surg Am. 2009;34(9): 1612-7. https://doi.org/10.1016/j.jhsa.2009.07.008.

7. Hershman $\mathrm{SH}$, et al. The effects of pronator quadratus repair on outcomes after volar plating of distal radius fractures. J Orthop Trauma. 2013;27(3): 130-3. https://doi.org/10.1097/BOT.0b013e3182539333.

8. Tosti R, llyas AM. Prospective evaluation of pronator quadratus repai following volar plate fixation of distal radius fractures. J Hand Surg Am. 2013:38(9):1678-84. https://doi.org/10.1016/j.jhsa.2013.06.006.

9. Häberle $S$, et al. Pronator quadratus repair after volar plating of distal radius fractures or not? Results of a prospective randomized trial. Eur J Med Res. 2015;20:93. https://doi.org/10.1186/s40001-015-0187-4.

10. Sonntag J, et al. No effect on functional outcome after repair of pronator quadratus in volar plating of distal radial fractures: a randomized clinical trial. Bone Joint J. 2019;101-b(12):1498-505. https://doi.org/10.1302/0301620x.101b12.Bjij-2019-0493.R1.

11. Liberati $A$, et al. The PRISMA statement for reporting systematic reviews and meta-analyses of studies that evaluate health care interventions: explanation and elaboration. J Clin Epidemiol. 2009;62(10):e1-34. https://doi.org/10.1016/ j.jclinepi.2009.06.006.

12. Jadad AR, et al. Assessing the quality of reports of randomized clinical trials: is blinding necessary? Control Clin Trials. 1996;17(1):1-12. https://doi.org/10. 1016/0197-2456(95)00134-4.

13. Stang A. Critical evaluation of the Newcastle-Ottawa Scale for the assessment of the quality of nonrandomized studies in meta-analyses. Eur J Epidemiol. 2010;25(9):603-5. https://doi.org/10.1007/s10654-010-9491-z.

14. Sun $L$, et al. Prognostic value of pathologic fracture in patients with high grade localized osteosarcoma: a systemic review and meta-analysis of cohort studies. J Orthop Res. 2015;33(1):131-9. https://doi.org/10.1002/jor. 22734.
15. JJ, D., H. JPT, and A. DG, Cochrane Handbook for Systematic Reviews of Interventions 10.10.2. 2nd ed. 2019: Chichester (UK): John Wiley \& Sons.

16. Sterne JA, Egger M. Funnel plots for detecting bias in meta-analysis: guidelines on choice of axis. J Clin Epidemiol. 2001;54(10):1046-55. https:// doi.org/10.1016/s0895-4356(01)00377-8.

17. Peters $\mathrm{J}$, et al. Comparison of two methods to detect publication bias in meta-analysis. JAMA. 2006;295(6):676-80. https://doi.org/10.1001/jama.295.6. 676.

18. Hohendorff $B$, et al. Pronator quadratus repair with a part of the brachioradialis muscle insertion in volar plate fixation of distal radius fractures: a prospective randomised trial. Arch Orthop Trauma Surg. 2018; 138(10):1479-85. https://doi.org/10.1007/s00402-018-2999-5.

19. Chao $Y$, et al. The effect of repairing pronator quadratus after distal radius fracture plating surgery [in Chinese]. J Practical Orthopaedics. 2019;25(11): 1015-8. https://doi.org/10.13795/j.cnki.sgkz.2019.11.014.

20. Zhang $Y$, et al. Clinical effect of palmar locking plate combined with repair of pronator quadratus muscle in the treatment of distal radius fracture [In Chinese]. Medical Innovation of China. 2018;15(14):111-5. https://doi.org/10. 3969/j.issn.1674-4985.2018.14.027.

21. Si W, Qin W, Hao Y. Middle-term clinical effect of pronator quadratus after volar plate of a distal radius fracture [in Chinese]. J Pracitcal Orthopaedics. 2012;18(11):973-5.

22. Qian Z. Comparison the functional recovery of repairing pronator quadratus or not after internal fixation of distal radius fractures [in Chinese]. Chines Journal of Hand Surgery. 2017:33(2). https://doi.org/10.3760/cma.j.issn.1005054X.2017.02.027.

23. Pathak $\mathrm{S}$, et al. Do we really need to repair the pronator quadratus after distal radius plating? Chin J Traumatol. 2019;22(6):345-9. https://doi.org/10. 1016/j.cjtee.2019.10.002

24. Wang H, Li Q. Analysis of functional effect of repairing pronator quadratus muscle after operation for distal radius [in Chinese]. Shannxi Med J. 2018; 47(9):1164-70. https://doi.org/10.3969/j.issn.1000-7377.2018.09.020

25. Cang T, Li Y. The effect of repairing of the pronator quadratus on postoperative hand function druing open reduction and internal fixation of distal radius fractures [in Chinese]. Chinese Journal of Clinical Rational Drug Use. 2019;12(32):190-1. https://doi.org/10.15887/j.cnki.13-1389/r.2019.32.118.

26. Marsh $\mathrm{J}$, et al. Fracture and dislocation classification compendium - 2007 : Orthopaedic Trauma Association classification, database and outcomes committee. J Orthop Trauma. 2007;21(10 Suppl):S1-133. https://doi.org/10. 1097/00005131-200711101-00001.

27. Stuart PR. Pronator quadratus revisited. J Hand Surg Br. 1996;21(6):714-22. https://doi.org/10.1016/s0266-7681(96)80175-6.

28. Ahsan ZS, Yao J. The importance of pronator quadratus repair in the treatment of distal radius fractures with volar plating. Hand (N Y). 2012;7(3): 276-80. https://doi.org/10.1007/s11552-012-9420-6.

29. Chen $\mathrm{H}$, et al. Validation of the simplified Chinese (Mainland) version of the Disability of the Arm, Shoulder, and Hand questionnaire (DASH-CHNPLAGH) J Orthop Surg Res. 2015:10:76. https://doi.org/10.1186/s13018-015-0216-6.

30. Offenbacher $M$, et al. Validation of a German version of the 'Disabilities of Arm, Shoulder and Hand' questionnaire (DASH-G). Z Rheumatol. 2003;62(2): 168-77. https://doi.org/10.1007/s00393-003-0461-7.

31. Schonnemann JO, et al. Reliability and validity of the Danish version of the disabilities of arm, shoulder, and hand questionnaire in patients with fractured wrists. J Plast Surg Hand Surg. 2011;45(1):35-9. https://doi.org/10. 3109/2000656×.2011.554708

32. Lozano-Calderon SA, et al. Wrist mobilization following volar plate fixation of fractures of the distal part of the radius. J Bone Joint Surg Am. 2008;90(6): 1297-304. https://doi.org/10.2106/JBJS.G.01368.

33. Asadollahi S, Keith PP. Flexor tendon injuries following plate fixation of distal radius fractures: a systematic review of the literature. J Orthop Traumatol. 2013;14(4):227-34. https://doi.org/10.1007/s10195-013-0245-z.

34. Thorninger $\mathrm{R}$, et al. Complications of volar locking plating of distal radius fractures in 576 patients with 3.2 years follow-up. Injury. 2017:48(6):1104-9. https://doi.org/10.1016/j.injury.2017.03.008.

\section{Publisher's Note}

Springer Nature remains neutral with regard to jurisdictional claims in published maps and institutional affiliations. 